\title{
Análise Retrospectiva de Pacientes Vítimas de Trauma Cervical Penetrante Submetidos À Cervicotomia
}

\author{
${ }^{1}$ Ana Cláudia Marchi Barros, ${ }^{2}$ Guilherme Damaceno Pereira, ${ }^{3}$ Marília França Madeira Manfrinato \\ ${ }^{4}$ Mariane Christina Savio, ${ }^{5}$ Camila de Souza Justini, ${ }^{6}$ Rafaela de Araujo Molteni \\ ${ }^{7}$ Fábio Henrique de Carvalho, ${ }^{8}$ Adonis Nasr
}

\begin{abstract}
RESUMO
Objetivos: As lesões cervicais penetrantes são, em sua maioria, causadas por armas de fogo ou armas brancas, e o conceito de um manejo cirúrgico seletivo é o empregado atualmente no tratamento destes pacientes. O presente trabalho tem como objetivos avaliar o perfil demográfico dos pacientes vítimas de traumas cervicais penetrantes submetidos à cervicotomia, quanto à presença ou não de lesão, o tipo de lesão encontrada durante o ato cirúrgico, além da evolução e mortalidade.
\end{abstract}

Métodos: Foram estudadas 57 vítimas de trauma cervical penetrante atendidas no Hospital do Trabalhador, em Curitiba (Brasil), nos períodos de abril de 2009 até março 2014, que foram submetidas à cervicotomia.

Resultados: Os principais mecanismos de trauma foram: ferimento por arma branca $(52,6 \%)$, ferimento por arma de fogo (40\%); $61 \%$ deles ocorreram em zona II. $91 \%$ dos pacientes eram do sexo masculino, com uma média de idade de 33,1 anos. $43,8 \%$ destes apresentavam alguma lesão associada e $46 \%$ realizaram exame de imagem pré-operatório. Em 82,5\% das cervicotomias foi encontrada lesão. As injúrias mais comuns foram: vascular (68\%), de vias aéreas (47\%) e de esôfago (11\%). O tempo operatório médio foi de 118 minutos. $17,5 \%$ dos pacientes apresentaram algum tipo de complicação pós-operatória. O tempo médio de internamento foi de 9 dias. Óbito ocorreu em $7 \%$ dos pacientes, sendo que todos estes apresentavam lesões associadas.

Conclusão: Homens adultos jovens são os mais acometidos. Todos os pacientes que foram à óbito apresentavam algum tipo de lesão associada, mostrando que a cervicotomia foi terapêutica quando existiam lesões cervicais isoladas. No entanto, em uma parte $(17,5 \%)$ dos pacientes submetidos à cirurgia, nenhuma lesão foi encontrada. Isto mostra que, apesar dos avanços tecnológicos referentes aos exames complementares, mais estudos são necessários para orientar

\footnotetext{
${ }^{1-5}$ Medical Student, ${ }^{6}$ General Surgeon, ${ }^{7,8}$ Trauma Surgeon and Professor

${ }^{1-8}$ Department of General and Trauma Surgery, Hospital do Trabalhador - Universidade Federal do Paraná (UFPR) Curitiba, Paraná, Brazil
}

Corresponding Author: Ana Cláudia Marchi Barros, Medical Student at Universidade Federal do Paraná, Avenida João Gualberto, 1313 ap 30580030001 , Curitiba, Paraná, Brasil e-mail: anaclaudiamarchibarros@gmail.com a conduta frente ao paciente vítima de trauma cervical penetrante.

Palavras claves: Cervicotomia, Hard signs, Trauma cervical.

How to cite this article: Barros ACM, Pereira GD, Manfrinato MFM, Savio MC, de Souza Justini C, de Araujo Molteni R, de Carvalho FH, Nasr A. Análise Retrospectiva de Pacientes Vítimas de Trauma Cervical Penetrante Submetidos À Cervicotomia. Panam J Trauma Crit Care Emerg Surg 2015; 4(2):96-102.

\section{Source of support: $\mathrm{Ni}$}

Conflict of interest: None

\section{ABSTRACT}

Objectives: Penetrating neck injuries are, for the majority caused by firearms or knives and the concept of a selective surgical management is the current approach in treating these patients. The present work aims to evaluate the demographic profile of patients suffering from cervical penetrating trauma for the presence or absence of injury, the type of injury found during surgery, plus the outcomes.

Materials and methods: We studied 57 patients with cervical trauma penetrating treated at Hospital worker in Curitiba (Brazil), in the period April 2009 to March 2014.

Results: The main trauma mechanisms were: stab wound $(52.6 \%)$, injury by firearm (40\%); $61 \%$ of them occurred in zone II. $91 \%$ of patients were male, with an average age of 33.1 years. $43.8 \%$ of them had some associated injury. $46 \%$ underwent preoperative imaging. In $82.5 \%$ who had surgery injury was found. Common were: vascular (68\%), airway (47\%) and esophagus (11\%). Mean operative time was 118 minutes. $17.5 \%$ of patients had some type of complication postoperatively. The average time of hospitalization was 9 days. Death occurred in $7 \%$ of patients, all of them had associated injuries.

Conclusion: Men young adults are the most affected. All patients who died presented some type of injury associated. Operation was therapeutic when there were isolated cervical lesions. However, in $17.5 \%$ of patients undergoing surgery, no lesion was found. More studies are needed to guide the management of patients with cervical penetrating trauma.

Keywords: Cervical trauma, Cervicotomy, Hard signs.

\section{INTRODUÇÃO}

A região cervical é muito vulnerável tanto pela ausência de proteção óssea como por sua anatomia, a qual reúne

Note: Paper presented in Student Research Competition at the Annual Congress of Panamerican Trauma Society, Panama City, Panama, November 2015. 
muitas estruturas vitais, incluindo traqueia, esôfago, tireoide e vasos sanguíneos calibrosos. Traumas desta região podem causar lesões graves com alta morbimortalidade. ${ }^{1}$

O trauma penetrante de pescoço pode ser causado por ferimentos por armas de fogo (FAF), por armas brancas (FAB) ou por outros detritos penetrantes (vidro e estilhaços). Consideram-se ferimentos penetrantes os que ultrapassam o músculo platisma. Quando o platisma encontra-se intacto a lesão é considerada superficial.

Até a década de 40 , todas as lesões penetrantes de pescoço eram tratadas de modo conservador, com taxas de mortalidade chegando aos 35\%. ${ }^{3}$ Em uma tentativa de reduzir as elevadas taxas de mortalidade, a abordagem cirúrgica foi adotada, inclusive para os pacientes estáveis.

Esta nova conduta gerou queda na taxa de mortalidade, mas também um incremento no número de explorações cirúrgicas negativas. Além disso, após a guerra, com o avanço dos métodos diagnósticos de imagem e com a predominância de lesões menos severas, surgiu o conceito do manejo cirúrgico seletivo, tendo como ponto de apoio o emprego de exames complementares radiológicos e endoscópicos.

Essa série de mudanças levou a uma quebra de paradigma no manejo de lesões cervicais penetrantes. Avanços contínuos também no manejo perioperatório e em anestesia contribuíram para melhores resultados no tratamento das lesões. ${ }^{2}$

O manejo da lesão cervical penetrante depende do estado hemodinâmico do paciente e do nível anatômico da injúria. ${ }^{4} \mathrm{O}$ pescoço é dividido em três zonas: I, II e III (Figura 1). A zona I vai das clavículas ou esterno até a borda inferior da cartilagem cricoide; a zona II vai da

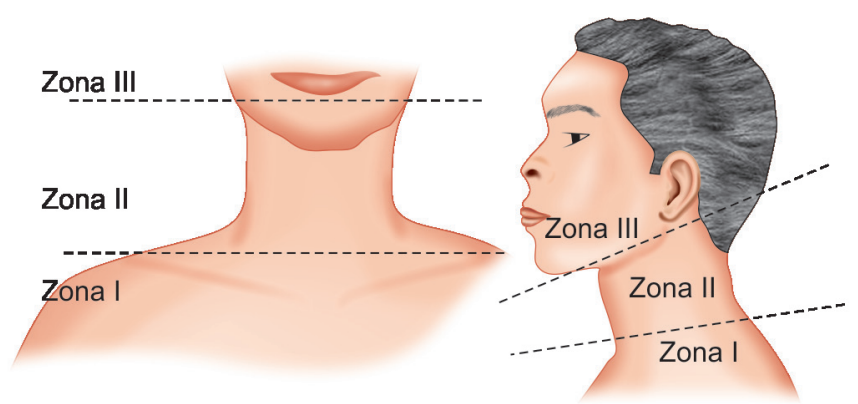

Figura 1: Divisão anatômica da região cervical ${ }^{1}$

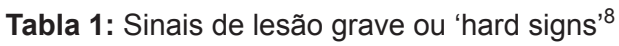

Comprometimento de via aérea

Enfisema subcutâneo massivo/escape de ar pela ferida

Hematoma pulsátil ou em expansão

Sangramento ativo

Choque

Déficit neurológico

Hematêmese cricoide ao ângulo da mandíbula e a zona III do ângulo da mandíbula até a base do crânio. ${ }^{5,6} \mathrm{~A}$ borda posterior do músculo esternocleidomastoideo é usada como divisão de pescoço anterior e posterior.

Manifestações clínicas significativas de lesões de estruturas importantes são: disfagia (traqueia e/ou esôfago); rouquidão (nervo laríngeo recorrente, laringe); sangramento orofaríngeo, nasofaríngeo ou ambos (lesão vascular); déficit neurológico e hipotensão arterial (não específico). $^{2}$

Na presença de instabilidade hemodinâmica ${ }^{4,7}$ ou dos chamados 'hard signs', isto é, sinais indicativos de lesão grave, a transferência para a sala de cirurgia deve ser imediata, com eventual realização de procedimentos necessários para garantia de via aérea definitiva e/ou controle de sangramento ativo importante ainda na sala de emergência (Tabla 1). ${ }^{8}$

O presente trabalho tem como objetivo avaliar o perfil epidemiológico e características das lesões das vítimas de trauma cervical penetrante que foram submetidas à cervicotomia em um centro de trauma.

\section{MÉTODOS}

Foi realizada a análise restrospectiva de prontuários de pacientes vítimas de trauma atendidas no pronto socorro do Hospital do Trabalhador, local de referência em atendimento de trauma, localizado em Curitiba-PR (Brasil), no período de $1^{\circ}$ de abril de 2009 a 31 de marco de 2014.

Olevantamento dos prontuários foi realizado por meio da busca no sistema eletrônico Hospub dos procedimentos 'tratamento cirúrgico de lesões traumáticas da região cervical', 'traqueorrafia', 'esofagorrafia' e 'laringorrafia'. Foram selecionados apenas os pacientes vítima de trauma cervical penetrante, sendo excluídos as vítimas de trauma contuso e os pacientes submetidos aos procedimentos acima por causas não traumáticas, com um total de 57 pacientes.

Os dados analisados foram identificação do paciente, sexo, idade, mecanismo de trauma (arma de fogo ou arma branca), topografia da ferida (zona cervical I, II ou III), sinais e sintomas presentes na admissão (enfisema subcutâneo, hematoma em expansão, rouquidão, hemoptise, sangramento ativo e choque hipovolêmico), realização de exames de imagem préoperatórios, lesão encontrada durante o ato operatório, tempo cirúrgico, necessidade de internamento em Unidade de Terapia Intensiva, tempo de internamento e desfecho do caso.

Os dados foram incluídos em uma Tabla Excel Microsoft ${ }^{\circledR}$ e apresentados na forma de estatística descritiva. 


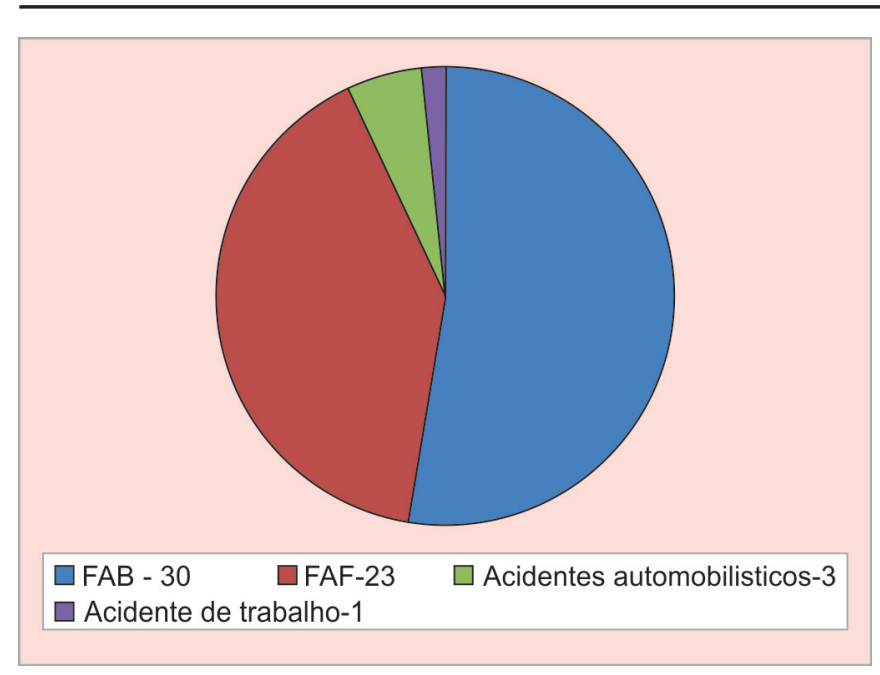

Gráfico 1: Mecanismos de trauma dos pacientes do estudo

\section{RESULTADOS}

Foram incluídos no estudo um total de 57 pacientes, com idade média de 33,1 anos (9-65 anos), sendo 91\% do sexo masculino. O mecanismo de trauma mais comum foi por arma branca, sendo 30 pacientes, responsáveis por $52,6 \%$ dos casos; seguidos pelos ferimentos de arma de fogo em 23 pacientes, sendo $40 \%$ dos casos; os por estilhaço de vidro durante acidentes automobilísticos somam 3 pacientes, o equivalente a 5,3\% das vítimas e 1 caso $(1,7 \%)$ vítima de trauma direto por objeto de trabalho (Gráfico 1).

Em relação à localização do ferimento, a mais frequentemente lesada foi a zona II, em $35(61,4 \%)$ das 57 vítimas. A seguir, a zona III, foi lesada em 3 casos (5,3\%). Nenhum paciente foi atendido com lesão isolada em zona I. Dois $(3,4 \%)$ pacientes apresentaram lesão em zona I, sendo um caso associado à lesão em zona II e outro à lesão em zona III. Associação de lesões em zona II e III foi encontrada em 4 casos (7\%). Em 12 (21\%) prontuários não foram encontradas informações a respeito da zona cervical atingida.

Dos 57 pacientes, 12 (21\%) foram admitidos hemodinamicamente instáveis, 5 (42\%) destes após resposta a reposição volêmica tornaram-se estáveis e foram enviados à angiotomografia cervical e, em função dos achados do exame, encaminhados ao centro cirúrgico. Os outros 7 (58\%) não responderam à reposição volêmica sendo prontamente encaminhados à cervicotomia, sem a realização de exames complementares. Dos 12 pacientes

Tabla 2: Pacientes com sinais sugestivos de lesão grave (hard signs)

\begin{tabular}{lll}
\hline Enfisema subcutâneo & 16 & $28 \%$ \\
Hematoma em expansão & 2 & $3,50 \%$ \\
Choque & 12 & $21 \%$ \\
Sangramento ativo & 23 & $40,30 \%$ \\
Disfonia & 4 & $7 \%$ \\
Exposição de tireoide & 1 & $1,70 \%$ \\
Escape de ar pela lesão & 1 & $1,70 \%$ \\
\hline
\end{tabular}

admitidos em choque hipovolêmico, 5 apresentaram lesão vascular à cervicotomia-o que justificava tal achado no exame (2 deles com lesão em carótida, outros 2 em veia jugular externa e 1 em veia jugular interna), 3 apresentavam lesão em vias aéreas e a instabilidade hemodinâmica pode ser explicada pelos ferimentos associados e, os outros 4 apresentaram cervicotomia não terapêutica, sendo o choque hipovolêmico em função das lesões associadas. Nenhum dos pacientes do grupo hemodinamicamente instáveis e em que a cervicotomia foi não terapêutica realizaram exames de imagem préoperatórios.

A maioria dos pacientes atendidos, $79 \%$ (45 pacientes), encontravam-se estáveis hemodinamicamente e foram submetidos à cirurgia em função de dados positivos no exame físico na admissão (64\%), reavaliações (13\%) ou em exames de imagem. $47 \%$ destes (21 pacientes) foram submetidos à angiotomografia cervical antes do procedimento cirúrgico.

Em 40 pacientes $(70 \%)$, achados de exame físico foram sugestivos de lesão: enfisema subcutâneo $(28 \%)$, hematoma em expansão $(3,5 \%)$, choque $(21 \%)$, sangramento ativo $(40,3 \%)$, disfonia $(7 \%)$, exposição de tireoide $(1,7 \%)$ e escape de ar pela lesão $(1,7 \%)$. Dos pacientes com choque hipovolêmico, apenas $1(1,7 \%)$ apresentava ferimento cervical isolado (Tabla 2).

Dos 57 pacientes, $25(43,8 \%)$ apresentaram lesões associadas, sendo que $10(40 \%)$ destes sofreram outros FAF, 9 (36\%) outros FAB, 5 (25\%) fratura óssea, $2(8 \%)$ traumatismo crânioencefálico e outros $2(8 \%)$ ferimentos corto-contusos em membros ou face. Lembrando que alguns pacientes sofreram mais que um tipo de lesão associada.

O tempo médio de cirurgia foi de 118 minutos, sendo que esse tempo inclui o de procedimentos complementares. Quando apenas a cervicotomia foi realizada, o tempo cirúrgico médio foi de 98 minutos.

A cervicotomia foi não terapêutica em 10 pacientes $(17,5 \%)$, sendo que em quatro destes foi realizada angiotomografia cervical préoperatória.

Em relação às lesões cervicais encontradas durante a cirurgia (Tabla 3), a mais comum foi a vascular, encontrada em 32 pacientes (68\%), seguida das lesões de vias aéreas, em 22 casos (46,8\%); lesões esofágicas, em 5 casos (10,6\%); lesões de tireoide, em 4 casos $(8,5 \%)$ e 3 casos $(6,3 \%)$ de lesão nervosa.

Tabla 3: Lesões encontradas durante cervicotomia

\begin{tabular}{lll}
\hline Vascular & 32 & $68 \%$ \\
Vias aéreas & 22 & $46,80 \%$ \\
Esôfago & 5 & $10,60 \%$ \\
Tireoide & 4 & $8,50 \%$ \\
Sistema nervoso & 3 & $6,30 \%$ \\
\hline
\end{tabular}


As lesões vasculares foram de veia jugular interna em $15(46,8 \%)$ pacientes; veia jugular externa em $14(43,7 \%)$; artéria carótida em 2 (6,2\%); artéria vertebral em $1(3,1 \%)$; artéria submandibular em 1 (3,1\%); artéria lingual em 1 $(3,1 \%)$; artéria facial em $1(3,1 \%)$ e artéria tireoidiana em $1(3,1 \%)$. Um dos paciente apresentava fratura do osso hioide associada.

A taxa de morbidade foi de $17,5 \%$, com complicações presentes em 10 pacientes. A paralisia de pregas vocais ocorreu em $5(50 \%)$ casos, a fístula traqueoesofágica esteve presente em $3(30 \%)$ pacientes; alterações neurológicas em $4(40 \%)$ pacientes e $1(10 \%)$ caso de pseudoaneurisma de carótida.

O tempo médio de internamento foi de nove dias, sendo de 4,5 dias nos pacientes vítimas de trauma cervical isolado. Dos 57 pacientes, 24 (42,1\%) necessitaram de cuidados em unidade de terapia intensiva, sendo que metade (12) deles apresentavam lesões associadas. Três (7\%) pacientes evoluíram para óbito, todos com lesões associadas, sendo o êxito letal em dois deles decorrente de choque hipovolêmico e em outro, morte encefálica por TCE grave associado.

\section{DISCUSSÃO}

Os traumatismos da região cervical se destacam por sua elevada complexidade e alta morbidade. Não há no corpo outro segmento que contenha estruturas representativas de tantos sistemas em um espaço tão confinado como o pescoço: sistemas respiratório, digestivo, vascular, nervoso central e periférico, linfático e endócrino.

As lesões penetrantes de pescoço correspondem a 5 a 10\% dos casos de trauma na emergência e apresentam o mesmo perfil de vítimas de trauma em geral—homens jovens. Neste estudo os pacientes apresentaram idade média de 33,1 anos, sendo $91 \%$ do sexo masculino. A incidência de zona lesada foi de II $(61,4 \%)$, seguida da III $(5,3 \%)$ e da I (3,4\%), similar à apresentada na literatura.

A abordagem do trauma cervical penetrante mudou substancialmente no decorrer das últimas décadas, da exploração cirúrgica mandatória ao tratamento conservador. O manejo inicial é decisivo no sucesso do tratamento do paciente. Deve-se considerar a mortalidade oriunda de lesões vasculares graves, que chega a $50 \%$, e as complicações tardias, como pseudoaneurismas e fístulas artério-venosas, que podem afetar os resultados em longo prazo. Assim sendo, o manejo correto, no momento adequado, é fundamental.

De acordo com o Western Trauma Association, a abordagem inicial deve seguir o guideline do ATLS (Advanced Life Trauma Support), com enfoque nas lesões que geram risco de morte imediato e com prioridade de tratamento.
Pacientes com estabilidade hemodinâmica e sem sinais de lesões graves podem ser manejados com uma conduta conservadora, através da realização de exames de imagem e exame físico seriado.

Na suspeita de lesão, o tratamento é determinado pela localização da lesão e pela situação hemodinâmica do paciente. ${ }^{5}$ Pacientes instáveis devem ser submetidos à cervicotomia exploradora.

Em pacientes hemodinamicamente estáveis, o local do ferimento irá determinar seu manejo.

Ferimentos em zona I (das clavículas até a cartilagem cricoide) devem receber avaliação de possíveis lesões vasculares, com angiotomografia de cervical e tórax; de vias aéreas, com fibrobroncoscopia e; do aparelho digestório, com endoscopia digestiva alta. Em caso positivo, deve-se proceder ao reparo da lesão, seja por cervicotomia exploradora ou por procedimentos endovasculares.

Pacientes com ferimentos em zona II, estáveis hemodinamicamente, e sem sintomas, podem ser observados sem necessidade de exames complementares. Quando se suspeita de lesão cervical, seja pela presença de enfisema subcutâneo, hematoma em região cervical, alterações vocais ou pelo trajeto do ferimento, deve-se realizar uma angiotomografia da região cervical. Neste caso, assim como em ferimentos da zona I, os achados do exame radiológico guiarão a conduta.

Por último, pacientes hemodinamicamente estáveis com ferimento de zona III devem ser submetidos à angiotomografia cervical e de crânio para investigação de danos vasculares e aerodigestivos. Lesões vasculares são manejadas com embolização ou colocação de 'stent', por meio de procedimentos endovasculares. $\mathrm{Na}$ suspeita de lesões de vias aéreas e/ou do tubo digestivo, os procedimentos endoscópicos são indicados, pois apresentam maior sensibilidade no seu diagnóstico em relação aos exames contrastados. ${ }^{9,10}$

Atualmente, o trauma cervical penetrante, apresenta uma taxa de mortalidade de 3 a $6 \%$, sendo muito menos que no passado- $11 \%$ na primeira guerra mundial. Neste estudo, a taxa de mortalidade, $7 \%$, foi similar à encontrada na literatura e decorrente parcialmente de lesões associadas.

A lesãos de grandes vasos (carótida, subclávia e jugular) é a principal causa de morte nos ferimentos penetrantes cervicais. Exsanguinação é a causa de morte mais comum e a estrutura mais comumente atingida é a artéria carótida 3,4, sendo afetada em 6 a 17\% dos pacientes. ${ }^{7,11}$ No presente estudo os vasos foram as estruturas mais frequentemente lesadas, especialmente as veias jugulares internas, externas e as carótidas, em $46 \%, 43 \%$ e $6 \%$ dos casos, respectivamente. 
As injúrias traumáticas dessa região englobam um grande número de lesões em adultos e continuam sendo de difícil manejo. ${ }^{3}$ Enquanto a intervenção cirúrgica é necessária em 15 a $20 \%$ dos casos (presença dos 'hard sings' ou de instabilidade hemodinâmica) a conduta frente ao paciente estável ainda é tema de discussão. ${ }^{3,8,12,13}$

Neste estudo, $79 \%$ dos pacientes se encontravam estáveis hemodinamicamente e foram submetidos à cervicotomia em função de dados do exame físico na admissão (64\%), de avaliações subsequentes (13\%) ou dos resultados de exames de imagem, ressaltando a importância do exame físico seriado.

Os exames de imagem não permitiram o desaparecimento das cervicotomias não terapêuticas, realizadas em dez pacientes nesse estudo, sendo que em 4 deles havia sido realizada angiotomografia cervical.

$\mathrm{O}$ fato de a interpretação dos exames de imagens ser feita pelo cirurgião do trauma e não por um radiologista, em grande parte dos casos, pode influenciar na decisão por exploração em casos com exames de imagem duvidosos. Apesar da morbidade associada à procedimentos cirúrgicos, a não realização de uma intervenção em pacientes com lesões potencialmente fatais é uma conduta associada à morbidade ainda maior.

Assim, em pacientes estáveis hemodinamicamente, deve-se levar em consideração o mecanismo de trauma e o trajeto dos ferimentos penetrantes, assim como a apresentação clínica e o resultado de exames de imagem para, em conjunto, optar-se ou não pela intervenção cirúrgica. ${ }^{8}$

Enquanto que no manejo de vítimas de trauma cervical com instabilidade hemodinâmica a cervicotomia exploradora tem seu papel bem estabelecido, em pacientes estáveis esta opção está em declínio em função do advento de técnicas endovasculares e dos avanços dos exames de imagem. No entanto, estudos Isto mostra que mais estudos são necessários para determinar critérios mais objetivos na indicação da cervicotomia exploradora nesses pacientes.

\section{REFERENCIAS}

1. Moore KL, Dalley AF. Anatomia orientada para clínica. 2006. Rio de Janeiro. Guanabara Koogan. 5. ed.

2. Alterman DM, Daley BJ, Selivanov V. Penetrating Neck Trauma. Medscape, 2008. Disponível em: http://emedicine. medscape.com/article/433306-overview\#a0112.

3. Thal ER, Meyer DM. Penetrating neck trauma. Curr Probl Surg 1992 Jan;29(1):1-56.

4. McConnell DB, Trunkey DD. Management of penetrating trauma to the neck. Adv Surg 1994;27:97-127.

5. Monson DO, Saletta JD, Freeark RJ. Carotid vertebral trauma. J Trauma 1969;9(12):987-999.

6. Roon AJ, Christensen N. Evaluation and treatment of penetrating cervical injuries. J Trauma 1979;19(6):391-397.

7. Demetriades D, Asensio JA, Velmahos G, Thal E. Complex problems in penetrating neck trauma. Surg Clin North Am 1996;(80):76-661.

8. Sperry JL, Moore EE, Coimbra R, et al. Western Trauma Association Critical Decisions in Trauma: penetrating neck trauma. J Trauma Acute Care Surg. Pittsburgh, Pennsylvania. 2013:75(6):936-940.

9. Offiah C, Hall E. Imaging assessment of penetrating injury of the neck and face. Insights Imaging 2012;3(5):419-431.

10. Ahmed N, Massier C, Tassie J, Whalen J, Chung R. Diagnosis of penetrating injuries of the pharynx and esophagus in the severely injured patient. J Trauma 2009;67(1):152-154.

11. Mittal VK, Paulson TJ, Colaiuta E, et al. Carotid artery injuries and their management. J Cardiovasc Surg (Torino) 2000;(3): 41-423.

12. Asensio JA, Valenziano CP, Falcone RE, Grosh JD. Management of penetrating neck injuries. The controversy surrounding zone II injuries. Surg Clin North Am 1991;(2):71-267.

13. Bryant AS, Cerfolio RJ. Esophageal trauma. Thorac Surg Clin 2007;17(1):63. 


\section{INVITED COMMENTARY}

La aproximación terapéutica del paciente con trauma cervical penetrante ha permanecido en constante cambio y evolución a través del tiempo. A mediados del siglo pasado, la mortalidad por trauma cervical penetrante alcanzaba cifras superiores al 30\%, lo que llevo a un ejercicio indiscriminado del manejo quirúrgico y como consecuencia a un aumento del número de exploraciones negativas que llego a alcanzar el $45 \%$ donde el denominador era la totalidad de los pacientes. Con el advenimiento de nuevos instrumentos y ayudas diagnosticas, se introdujo el concepto de cirugía selectiva con el fin de reducir el número de exploraciones negativas. En la actualidad, la propuesta es ser aun más selectivo que la cirugía selectiva, primero agotando los recursos y ayudas diagnosticas disponibles antes de operar al paciente.

El presente trabajo nos muestra la experiencia de 5 años de una institución hospitalaria en Curitiba-Brasil en el manejo del trauma cervical penetrante, y a través de ella podemos contrastar sus resultados con la actualidad en el manejo de esta entidad.

La tasa de exploraciones negativas reportadas en el estudio fue del 17,5\%, la cual a nuestro juicio continúa siendo elevada si se tiene en cuenta la cantidad de recursos diagnósticos disponibles en la actualidad. Sin embargo, no hay que desconocer la brecha que puede existir en la obtención, disponibilidad y oportunidad de los recursos diagnósticos por parte de algunas instituciones. Esta limitante puede ser aún más importante en países en vía de desarrollo (como la mayoría de los de la región) y por supuesto en zonas marginadas o de difícil acceso.

Adicional al elevado porcentaje de exploraciones negativas, se suman el de intervenciones quirúrgicas innecesarias. En este grupo se ubican aquellos pacientes llevados a cervicotomías con lesiones que probablemente hubieran podido recibir manejo no quirúrgico, porcentaje que también es elevado en el presente trabajo. Estos resultados, contrastan con las recomendaciones actuales donde se trata de evitar las intervenciones quirúrgicas innecesarias.

Teniendo en cuenta lo anterior, el enfoque del paciente con trauma cervical penetrante debe ser el siguiente: Pacientes inestables hemodinamicamente o con la presencia de algún 'signo duro' deben ser llevados a cirugía. Por otra parte, aquellos que no cumplan alguna de estas indicaciones, antes de considerar llevarlos a cirugía, se les debe realizar una arteriografía contrastada si se sospecha una lesión de la carótida; una laringoscopia/broncoscopia si la sospecha es de lesión en tráquea o un esofagograma contrastado y endoscopia si se sospecha compromiso esofágico. La tomografía axial computarizada (TAC) multicorte de cuello ha venido reemplazando estos exámenes, y hoy se ha convertido en el manejo diagnostico de primera línea, desplazando así a los exámenes anteriormente mencionados a ser ayudas diagnosticas de segunda línea exclusivamente cuando el TAC no logre ser conclusivo o aun existan dudas sobre la presencia de lesión. Es importante la observación y el seguimiento de estos pacientes mediante un examen físico exhaustivo el cual no debe ser reemplazado.

El advenimiento y los avances en la realización de procedimientos endovasculares, han posicionado esta rama de la medicina como una alternativa en el manejo de los pacientes con trauma cervical penetrante. Los hallazgos encontrados en el TAC serán el punto de partida para definir que pacientes se benefician de esta opción terapéutica, y con esto evitar de nuevo intervenciones quirúrgicas innecesarias. Los avances en esta disciplina sumada a la rapidez con la que se están obteniendo las imágenes del TAC, permiten incluso que pacientes hemodinamicamente inestables puedan ser llevados a la realización del TAC e incluso posterior a ello brindarles manejo endovascular.

Adoptar las recomendaciones actuales en el manejo del trauma cervical penetrante contribuirá a que esta entidad logre mantener bajas tasas de mortalidad con menor número de exploraciones quirúrgicas negativas y de exploraciones quirúrgicas innecesarias.

Es importante conocer la epidemiologia institucional, local y regional del trauma. Para ello se han hecho esfuerzos por fortalecer los registros de trauma y bases de datos que permitan recolectar esta información y poder tomar decisiones acertadas basadas en datos propios. En el capítulo de trauma cervical penetrante, trabajos como este ayudan a conocer en detalle los mecanismos y características del trauma, así como su manejo y desenlaces, que nos ayudan a retroalimentar nuestra práctica clínica y así detectar potenciales oportunidades de mejora. Se debe estimular y promover la realización de más estudios, cada vez más amplios, que caractericen estos perfiles demográficos y contribuyan a realizar cambios y acciones en nuestra práctica clínica.

Professor Carlos Ordonez

Jefe del Departamento de Cirugía. Universidad del Valle, Cali, Colombia Cirujano de Trauma y Emergencias. Hospital Unuversitario del valle, Fundación Valle del Lili, Cali, Colombia 
The therapeutic approach of patients with penetrating wounds to the neck has been in continuous change and evolution over time. In the middle of the last century, mortality rate from penetrating cervical trauma reached more than $30 \%$, which led to an indiscriminate exercise of surgical management due to an increased number of negative explorations $(45 \%)($ denominator $=$ all patients). With the introduction of new tools and diagnostic imaging, the concept of selective surgery was introduced in order to reduce the number of negative explorations. Now, the proposal is to be even more selective than selective surgery, using the available diagnostic resources before operating on the patient.

This paper shows a 5-year experience of a hospital in Curitiba, Brazil in the management of penetrating cervical trauma, and through it we can compare their results with today's guidelines in the management of this condition.

The negative exploration rate reported in the study was $17.5 \%$, which in our view remains high, if you consider the amount of diagnostic resources available. However, we must not ignore the gap that may exist in the obtaining, availability and promptness of diagnostic resources by some institutions. This limitation may be even more important in developing countries (like most of the region) and of course in remote areas.

In addition to the high percentage of negative explorations, there is the problem of unnecessary surgeries. This group of unnecessary surgeries is composed of patients who had cervical exploration for injuries that probably did not require surgical management. The percentage of this group, is high in the present study, in contrast to the current recommendations to avoid unnecessary surgeries.

The approach to patients with penetrating wounds to the neck must be: hemodynamic instability or the presence of a 'hard sign' as indications for the operating room. Those that do not meet any of these criteria, before surgery, should undergo a computed tomography (CT) arteriography if a carotid injury is suspected; a laryngoscopy/bronchoscopy if a trachea injury is suspected or an esophagogram and esophageal endoscopy if an esophageal injury is suspected. Cervical multislice CT has begun to replace these tests, and today is considered to be the first-line diagnostic tool, thus displacing the above tests to a second-line alternative, only when the CT fails to be conclusive or if there is doubt about the presence of any injury. It is important to observe and supervise patients through a comprehensive physical examination which should not be neglected.

The introduction and development of endovascular procedures have positioned this branch of medicine as an alternative in the management of patients with penetrating cervical trauma. Computed tomography findings will be the starting point for defining which patients may benefit from this alternative and thereby avoid unnecessary surgeries. Discipline development and rapid CT scanning, allow us even to take hemodynamically unstable patients to the scanner suite and bring them for an endovascular management.

These recommendations in the current management of penetrating cervical trauma may contribute to a lower mortality rate with fewer negative explorations and unnecessary surgical explorations.

It is important to know the institutional, local and regional epidemiology of trauma. To this end, efforts have been made to strengthen trauma registries and databases that allow to collect this information and help decision making. In penetrating cervical trauma. Work like this manuscript helps to know in detail trauma mechanisms and characteristics, as well as their management and outcomes, and help us to feedback our clinical practice and thus identify potential improvement opportunities. It should encourage and promote further studies, ever wider, which characterize these demographic profiles and contribute to make changes in our current clinical practice.

Professor Carlos Ordonez

Jefe del Departamento de Cirugía. Universidad del Valle, Cali, Colombia

Cirujano de Trauma y Emergencias. Hospital Unuversitario del valle, Fundación Valle del Lili, Cali, Colombia 